\title{
High nuclear karyopherin $\alpha 2$ expression is a strong and independent predictor of biochemical recurrence in prostate cancer patients treated by radical prostatectomy
}

Katharina Grupp ${ }^{1,2,5}$, Mareike Habermann ${ }^{2,5}$, Hüseyin Sirma ${ }^{2}$, Ronald Simon ${ }^{2}$, Stefan Steurer ${ }^{2}$, Claudia Hube-Magg², Kristina Prien ${ }^{2}$, Lia Burkhardt ${ }^{2}$, Karolina Jedrzejewska ${ }^{2}$, Georg Salomon ${ }^{3}$, Hans Heinzer ${ }^{3}$, Waldemar Wilczak ${ }^{2}$, Martina Kluth ${ }^{2}$, Jakob R Izbicki ${ }^{1}$, Guido Sauter ${ }^{2}$, Sarah Minner ${ }^{2}$, Thorsten Schlomm ${ }^{3,4}$ and Maria Christina Tsourlakis ${ }^{2}$

${ }^{1}$ General, Visceral and Thoracic Surgery Department and Clinic, Hamburg, Germany; ${ }^{2}$ Institute of Pathology, Hamburg, Germany; ${ }^{3}$ Martini-Clinic, Prostate Cancer Center, Hamburg, Germany and ${ }^{4}$ Department of Urology, Section for translational Prostate Cancer Research, University Medical Center Hamburg-Eppendorf, Hamburg, Germany

\begin{abstract}
Increased levels of karyopherin $\alpha 2$ (KPNA2) expression have been described to be linked to poor prognosis in a variety of malignancies. This study was undertaken to evaluate the clinical impact of KPNA2 expression and its association with key genomic alterations in prostate cancers. A tissue microarray containing samples from 11152 prostate cancers was analyzed for KPNA2 expression by immunohistochemistry. Results were compared with oncological follow-up data and genomic alterations such as TMPRSS2-ERG fusions and deletions of PTEN, $5 q 21,6 q 15$ or 3p13. KPNA2 expression was absent or weak in benign prostatic glands and was found to be in weak, moderate or strong intensities in $68.4 \%$ of 7964 interpretable prostate cancers. KPNA2 positivity was significantly linked to the presence of $E R G$ rearrangement $(P<0.0001)$. In ERG-negative and -positive prostate cancers, KPNA2 immunostaining was significantly associated with advanced pathological tumor stage (pT3b/ pT4), high Gleason grade and early biochemical recurrence $(P<0.0001$ each). Multivariate analysis including all established prognostic criteria available after surgery revealed that the prognostic role of KPNA2 $(P=0.001)$ was independent of high Gleason grade, advanced pathological tumor stage, high preoperative prostatespecific antigen level and positive surgical margin status $(P<0.0001$ each). The comparison of KPNA2 expression with deletions of PTEN, 5q21, 6q15 and 3p13 in ERG-positive and -negative cancers revealed a strong link to PTEN deletions in both subgroups $(P<0.0001)$. In conclusion, the strong independent prognostic impact of KPNA2 expression raises the possibility that measurement of KPNA2 expression alone or in combination with other molecular parameters might possibly result in clinically useful information. The data also emphasize a critical role of the functionality of the nuclear import machinery for prostate cancer biology. Modern Pathology (2014) 27, 96-106; doi:10.1038/modpathol.2013.127; published online 26 July 2013
\end{abstract}

Keywords: KPNA2; 3p13; prostate cancer; PTEN; 6q15; 5q21; tissue microarray

Prostate cancer is the most common malignancy in men in western societies. ${ }^{1}$ Even though a considerable proportion of prostate cancers has a rather indolent course, prostate cancer represents a major

Correspondence: Dr MC Tsourlakis, MD, Institute of Pathology, University Medical Center Hamburg-Eppendorf, Martinistrasse 52, 20246 Hamburg, Germany.

E-mail: mtsourlakis@uke.de

${ }^{5}$ These authors contributed equally to this work.

Received 5 December 2012; revised 19 May 2013; accepted 30 May 2013; published online 26 July 2013 cause of cancer-related death in men. ${ }^{1}$ Despite recent advantages in research, the only established pretreatment prognostic parameters currently include Gleason grade, tumor extent on biopsies, preoperative prostate-specific antigen (PSA) and clinical parameters. These data are statistically powerful but not sufficient for optimal individual treatment decisions. It can be hoped that the analysis of molecular features may enable a better individual prediction of tumor aggressiveness in the future.

Karyopherins are soluble nuclear transport receptors utilizing the nuclear pore complex for 
transporting cargo proteins and certain RNAs in and out of the cell nucleus. ${ }^{2}$ Karyopherin $\alpha 2$ (KPNA2) belongs to the karyopherin family and has been described to have an oncogenic role through the modulation of the subcellular localization of cargo proteins relevant for cancer. ${ }^{3}$ KPNA2 is also suspected to be involved in cellular proliferation, differentiation, cell-matrix adhesion, colony formation and migration. ${ }^{4-7}$ Increased KPNA2 expression levels, as compared with normal tissue, have been described in various malignancies including breast cancer, ${ }^{8}$ melanoma, ${ }^{9}$ ovarian cancer $^{10}$ and astrocytoma. ${ }^{11}$

Upregulation of nuclear KPNA2 protein expression was described in prostate cancer tissue and KPNA2 expression levels were found to be associated with PSA recurrence after radical prostatectomy in a cohort of 707 primary prostate cancer patients. ${ }^{4}$ In vitro experiments demonstrated that inhibition of KPNA2 reduced proliferation and viability of PC3 cells. ${ }^{4}$ In consequence of the detection of the TMPRSS2:ERG fusion prostate cancer is divided into subsets of fusion-positive and -negative tumors, which are linked to other genomic alterations, including deletions of PTEN and 3p13 in fusion-positive as well as deletions of 5q21 and 6q15 in fusion-negative cancers. ${ }^{12-19}$ As these deletions have all been linked to poor patient prognosis, ${ }^{12-19}$ we hypothesize that the prognostic value of KPNA2 might be modified by the presence or absence of these alterations. To learn more about the potential clinical utility of KPNA2 protein analysis and its association with known key molecular alterations in prostate cancer, a tissue microarray (TMA) containing 11152 prostate cancer specimens with clinical follow-up and an attached molecular database was utilized. Our data demonstrate that high KPNA2 staining is a strong and independent prognostic marker in prostate cancer.

\section{Materials and methods}

\section{Patients}

Radical prostatectomy specimens were evaluable from 11152 patients undergoing surgery between 1992 and 2011 at the Department of Urology, and the Martini Clinics at the University Medical Center Hamburg-Eppendorf. Follow-up data were available for a total of 9628 patients with a median follow-up of 36.8 months (range: 1-228 months; Table 1). PSA values were measured following surgery and recurrence was defined as a postoperative PSA of $0.2 \mathrm{ng} /$ $\mathrm{ml}$ and increasing at first appearance. All prostate specimens were analyzed according to a standard procedure, including a complete embedding of the entire prostate for histological analysis. ${ }^{20}$ The TMA manufacturing process was described earlier in detail. ${ }^{21}$ All hematoxylin- and eosinstained histological sections from all prostatectomy
Table 1 Pathological and clinical data of the arrayed prostate cancers

\begin{tabular}{|c|c|c|}
\hline & \multicolumn{2}{|c|}{ No. of patients } \\
\hline & $\begin{array}{l}\text { Study cohort on } \\
\text { TMA }(\mathrm{n}=11152)\end{array}$ & $\begin{array}{c}\text { Biochemical relapse } \\
\text { among categories } \\
(\mathrm{n}=1824)\end{array}$ \\
\hline \multicolumn{3}{|c|}{ Follow-up (months) } \\
\hline Mean & 53.4 & - \\
\hline Median & 36.8 & - \\
\hline \multicolumn{3}{|l|}{ Age (years) } \\
\hline$<50$ & 318 & 49 \\
\hline $50-60$ & 2.768 & 460 \\
\hline $60-70$ & 6.548 & 1.081 \\
\hline$>70$ & 1.439 & 232 \\
\hline \multicolumn{3}{|c|}{ Pretreatment PSA $(\mathrm{ng} / \mathrm{ml})$} \\
\hline$<4$ & 1.407 & 142 \\
\hline $4-10$ & 6735 & 827 \\
\hline $10-20$ & 2159 & 521 \\
\hline$>20$ & 720 & 309 \\
\hline \multicolumn{3}{|c|}{$p T$ category (AJCC 2002) } \\
\hline pT2 & 7.370 & 570 \\
\hline рТ3а & 2.409 & 587 \\
\hline pT3b & 1.262 & 618 \\
\hline pT4 & 63 & 49 \\
\hline \multicolumn{3}{|c|}{ Gleason grade } \\
\hline$\leq 3+3$ & 2.859 & 193 \\
\hline $3+4$ & 1.565 & 573 \\
\hline $4+3$ & 6.183 & 849 \\
\hline$\geq 4+4$ & 482 & 208 \\
\hline \multicolumn{3}{|l|}{$p N$ category } \\
\hline pNo & 6.117 & 1.126 \\
\hline $\mathrm{pN}+$ & 561 & 291 \\
\hline \multicolumn{3}{|c|}{ Surgical margin } \\
\hline Negative & 8.984 & 1.146 \\
\hline Positive & 1.970 & 642 \\
\hline
\end{tabular}

Abbreviation: AJCC, American Joint Committee on Cancer.

Note: Numbers do not always add up to 11152 in the different categories because of cases with missing data.

specimens were reviewed for the purpose of this study and the tumors were marked on the slides. One 0.6-mm tissue core was punched from a preselected area of each tumor and transferred into a TMA. The punch site was selected to contain the highest possible fraction of tumor cells. The tissues were distributed among 24 TMA blocks, each containing 144-522 tumor samples. The presence or absence of cancer tissue was validated by immunohistochemical AMACR and 34BE12 analysis on adjacent TMA sections. For internal controls, each TMA block also contained various control tissues, including normal prostate tissue. The molecular database attached to this TMA contained results on ERG expression in 9628 ERG break-apart fluorescence in situ hybridization (FISH) analysis in 6106 (expanded from Minner et $\mathrm{al}^{22}$ ), and deletion status of 5q21 in $3037,{ }^{16}$ 6q15 in 3528 (expanded from Kluth et $a l^{15}$ ), PTEN in $6130^{17}$ and 3p13 in 1290 (unpublished data) tumors. 


\section{Immunohistochemistry}

Freshly cut TMA sections were immunostained on one day and in one experiment. The primary antibody specific for KPNA2 (polyclonal; rabbit, Abcam; cat. no. ab84440; at 1/150 dilution) was applied, slides were deparaffinized and exposed to heatinduced antigen retrieval for $5 \mathrm{~min}$ in an autoclave at $121^{\circ} \mathrm{C}$ in Tris-EDTA buffer (pH 9). Bound antibody was then visualized using the EnVision Kit (Dako). One experienced person analyzed all stainings (KG). KPNA2 expression was predominantly localized in the nucleus, with lower expression levels in the cytoplasm of the cells. Nuclear KPNA2 staining was evaluated according to the following scoring system: the staining intensity $(0,1+, 2+$ and $3+)$ and the fraction of positive tumor cells were recorded for each tissue spot. A final immunohistochemistry (IHC) score was built from these parameters as described previously: ${ }^{23-25}$ negative scores had complete absence of staining, weak scores had staining intensity of $1+$ in $\leq 70 \%$ of tumor cells or staining intensity of $2+$ in $\leq 30 \%$ of tumor cells; moderate scores had staining intensity of $1+$ in $>70 \%$ of tumor cells, staining intensity of $2+$ in $>30 \%$ but in $\leq 70 \%$ of tumor cells or staining intensity of $3+$ in $\leq 30 \%$ of tumor cells; and strong scores had staining intensity of $2+$ in $>70 \%$ of tumor cells or staining intensity of $3+$ in $>30 \%$ of tumor cells. Cytoplasmatic KPNA2 staining was rare, typically associated with high nuclear staining levels and was thus not considered for analysis.

\section{Statistics}

Statistical calculations were performed with the JPM 9 software (SAS Institute, Cary, NC, USA).

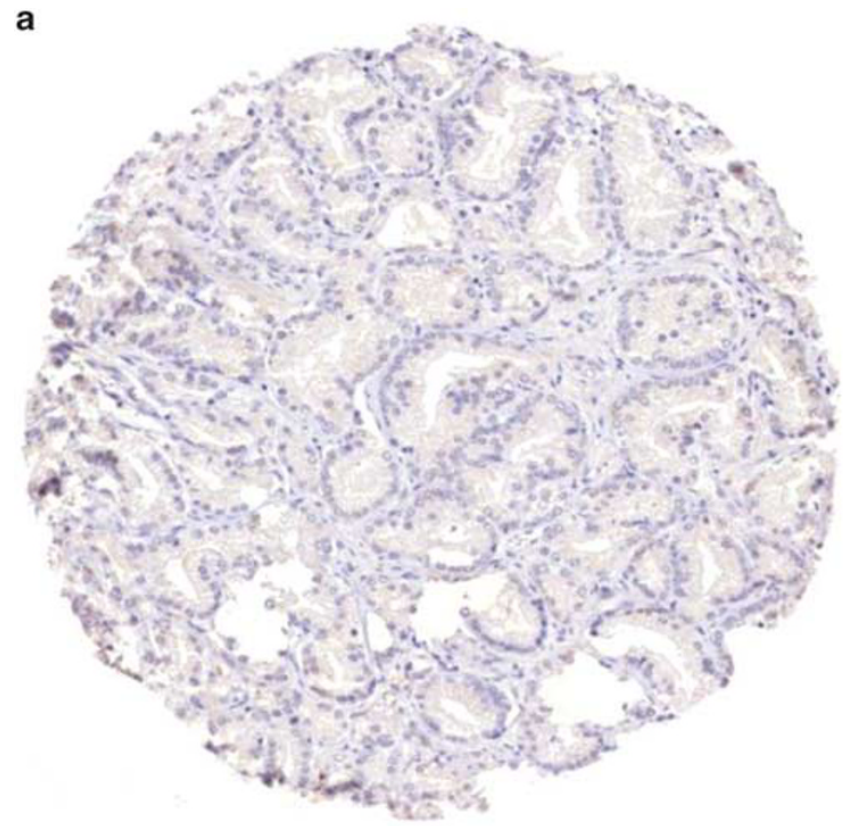

Contingency tables and the $\chi^{2}$ test were performed to search for associations between molecular parameters and tumor phenotype. Survival curves were calculated according to Kaplan-Meier. The log-rank test was applied to detect significant survival differences between groups. Cox proportional hazards regression analysis was performed to test the statistical independence and significance between pathological, molecular and clinical variables.

\section{Results}

\section{Technical Issues}

A total of 3188 of 11152 arrayed tissue samples $(28.6 \%)$ were non-informative for KPNA2 IHC because of the complete lack of tissue or absence of unequivocal cancer cells.

\section{IHC of KPNA2}

KPNA2 expression was found predominantly in the nucleus rarely accompanied by even lower staining levels in the cytoplasm of the cells. KPNA2 expression was absent or showed weak staining intensity in benign prostatic glands. The expression level of KPNA2 was thus higher in malignant prostate compared with benign tissue. In prostate carcinoma, positive KPNA2 staining was found in 5443 of our 7964 interpretable prostate cancers $(68.3 \%)$ and was considered weak in $32 \%$, moderate in $17.8 \%$ and strong in $18.5 \%$ of cases. Representative elements of a TMA stained with KPNA2 antibody are given in Figure 1.

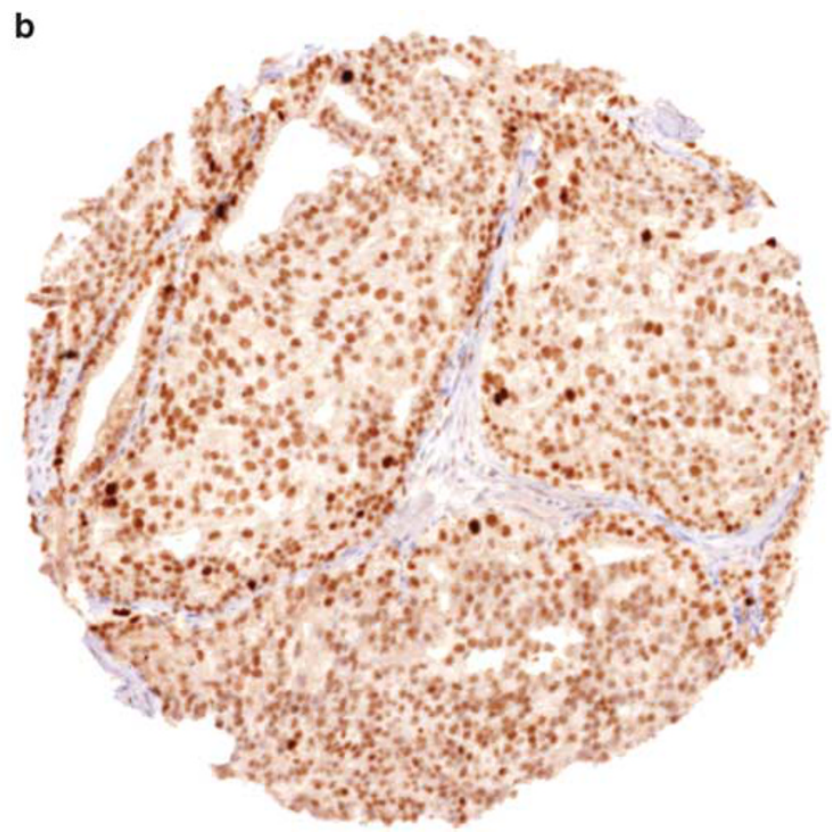

Figure 1 Representative elements of (a) absent and (b) strong KPNA2 expression in prostate cancer. 


\section{KPNA2 vs ERG Status}

To evaluate whether KPNA2 expression is linked to ERG status in prostate cancers, we took advantage of our TMA-attached database, including data on TMPRSS2-ERG fusion status obtained by FISH in 5036 tumors and by IHC in 7787 tumors for which KPNA2 staining was also available. Increased KPNA2 expression was strongly associated with the presence of TMPRSS2-ERG rearrangement and ERG protein expression $(P<0.0001$ each; Figure 2). Accordingly, the relationship between KPNA2 expression and histological phenotype and clinical cancer features was calculated separately for ERGpositive and -negative prostate cancer subsets (Tables 2 and 3). In both groups, high KPNA2 expression was significantly associated with unfavorable tumor phenotype. Increased KPNA2

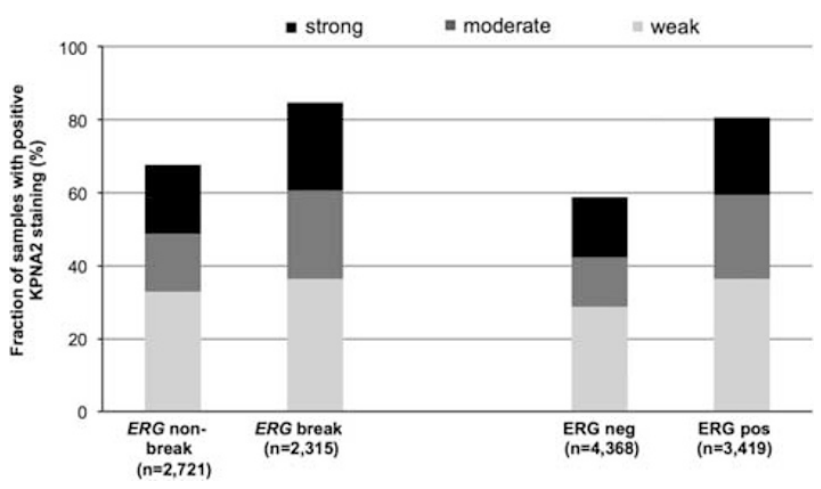

Figure 2 Relationship of KPNA2 expression with ERG status probed by fluorescence in situ hybridization analysis and immunohitochemistry $(P<0.0001$ each). expression was tightly linked to advanced tumor stage $(P<0.0001$ each $)$, high Gleason grade $(P<0.0001$ each $)$ and positive nodal involvement $(P<0.0001$ and $P=0.0325)$ (Tables 2 and 3$)$.

\section{Relationship with Other Key Genomic Deletions in ERG-Positive and -Negative Prostate Cancers}

Recent studies have provided evidence for distinct molecular subgroups of prostate cancers defined by TMPRSS2-ERG fusions and several genomic deletions. We and others have described a strong link of PTEN and 3p13 deletion to ERG positivity and of 5q21 and 6q15 deletions to ERG negativity. ${ }^{12-19}$ To evaluate whether KPNA2 staining might be particularly linked to one of these genomic deletions, immunohistochemical results were compared with pre-existing findings on PTEN, 3p13, 6q15 and 5q21 deletions. The analysis of all tumors demonstrated significant associations or at least strong trends of KPNA2 expression to these deletions (Figure 3). A subsequent subgroup analysis of ERG-positive and -negative cancers revealed that a strong association between KPNA2 expression and PTEN deletions was retained in both subgroups $(P<0.0001$; Figures 4 and 5). Within ERG-negative cancers, there was also a strong association of both $6 q 15$ and 5q21 deletions with high KPNA2 expression $(P<0.0001$ each; Figure 4$)$.

\section{Relationship with PSA Recurrence}

Follow-up data were available for 6853 patients with informative KPNA2 data. Strong KPNA2 staining was related to early biochemical recurrence in all

Table 2 Associations between KPNA2 expression results and ERG-negative prostate cancer phenotype

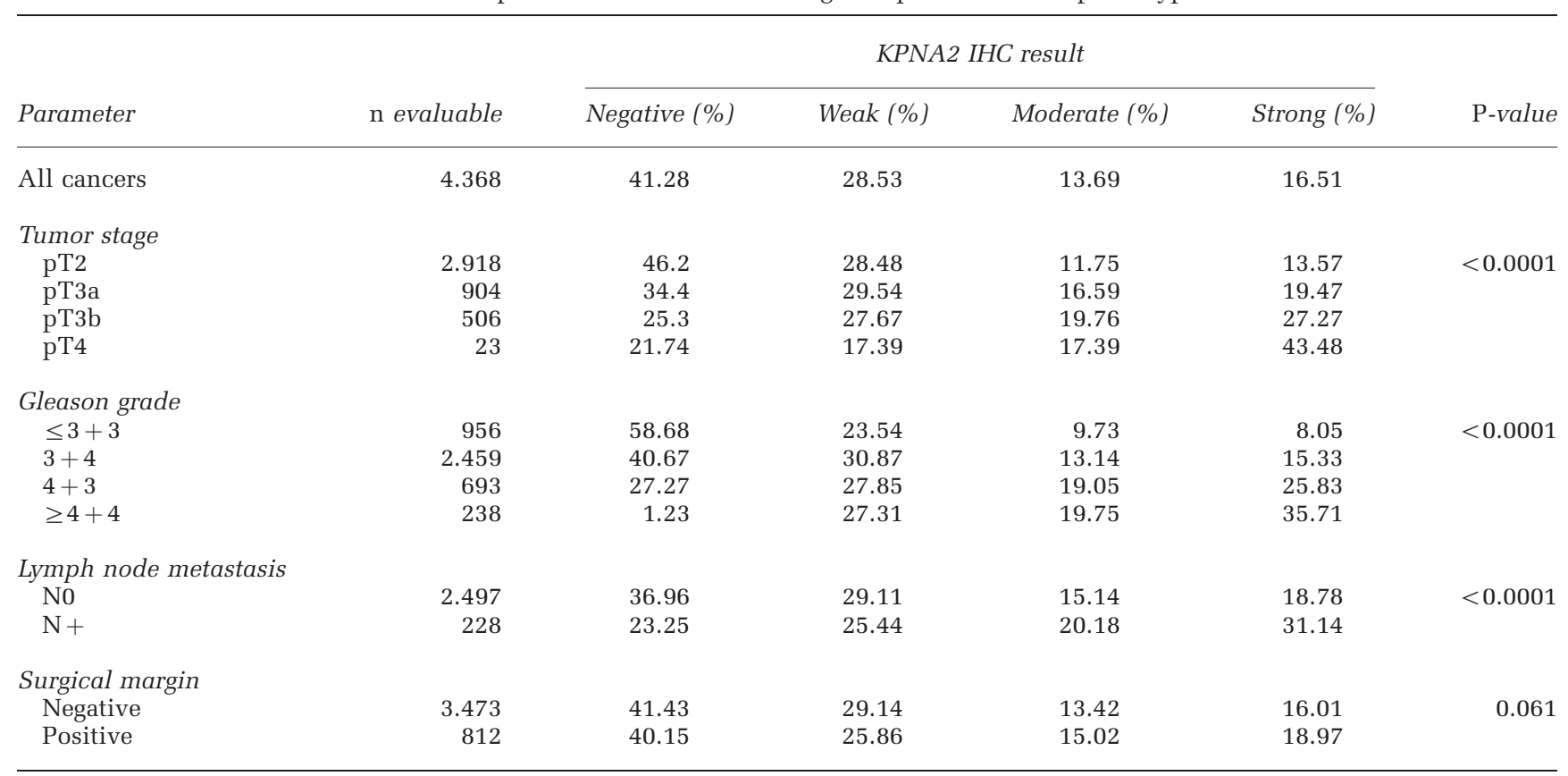


Table 3 Associations between KPNA2 expression results and ERG-positive prostate cancer phenotype

\begin{tabular}{|c|c|c|c|c|c|c|}
\hline \multirow[b]{2}{*}{ Parameter } & \multirow[b]{2}{*}{ n evaluable } & \multicolumn{4}{|c|}{ KPNA2 IHC result } & \multirow[b]{2}{*}{$\mathrm{P}$-value } \\
\hline & & Negative (\%) & Weak $(\%)$ & Moderate (\%) & Strong (\%) & \\
\hline All cancers & 3.419 & 19.07 & 36.44 & 23.11 & 21.38 & \\
\hline \multicolumn{7}{|l|}{ Tumor stage } \\
\hline pT2 & 2.076 & 21.34 & 38.2 & 22.93 & 17.53 & $<0.0001$ \\
\hline рТ3а & 896 & 15.85 & 34.38 & 23.66 & 26.12 & \\
\hline pT3b & 412 & 14.56 & 33.01 & 22.82 & 29.61 & \\
\hline pT4 & 20 & 20 & 20 & 10 & 50 & \\
\hline \multicolumn{7}{|c|}{ Gleason grade } \\
\hline$\leq 3+3$ & 773 & 28.46 & 37 & 19.92 & 14.62 & $<0.0001$ \\
\hline$\overline{3}+4$ & 2.043 & 17.03 & 37.49 & 24.33 & 21.15 & \\
\hline $4+3$ & 477 & 15.09 & 31.03 & 23.69 & 30.19 & \\
\hline$\geq 4+4$ & 105 & 7.62 & 36.19 & 17.14 & 39.05 & \\
\hline \multicolumn{7}{|c|}{ Lymph node metastasis } \\
\hline No & 1.888 & 15.2 & 36.39 & 24.63 & 23.78 & 0.0325 \\
\hline $\mathrm{N}+$ & 190 & 12.63 & 28.95 & 25.79 & 32.63 & \\
\hline \multicolumn{7}{|c|}{ Surgical margin } \\
\hline Negative & 2.710 & 19.45 & 37.16 & 22.36 & 21.03 & 0.1409 \\
\hline Positive & 652 & 18.25 & 33.44 & 25.61 & 22.7 & \\
\hline
\end{tabular}

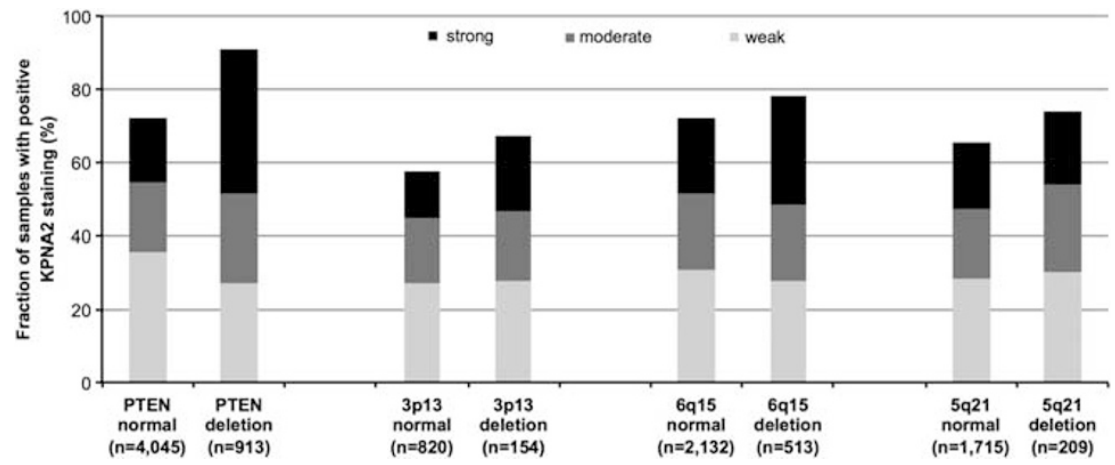

Figure 3 KPNA2 expression vs deletions at PTEN $(P<0.0001)$, 3p13 $(P=0.03)$, $6 q 15(P<0.0001)$ and $5 q 21(P=0.0537)$ probed by fluorescence in situ hybridization analysis in all prostate cancers.

cancers, as well as in the subsets of 3731 ERGnegative and 2960 ERG-positive cancers $(P<0.0001$ each; Figures $6 \mathrm{a}-\mathrm{C})$. A further analysis including the PTEN deletion status revealed that KPNA2 expression was still prognostically relevant in the subgroup of 3372 PTEN-non-deleted cancers $(P<0.0001$; Figure 6d). This significant association was retained in both PTEN-non-deleted ERG-negative and -positive tumors $(P<0.0001$ and $P=0.0009$; Figures 6e and f). The KPNA2 expression level did not provide additional prognostic information within the smaller subgroup of 798 PTEN-deleted cancers $(P=0.6082$; data not shown $)$.

\section{Multivariate Analysis}

Multivariate analysis including established prognostic criteria available after surgery revealed independent prognostic relevance for advanced Gleason grade, high tumor stage, high preoperative
PSA level and positive surgical margin status $(P<0.0001$ each), as well as for KPNA2 expression $(P=0.001)$. If the nodal status is included in multivariate analysis, the absolute number of analyzable patients is reduced from 6691 to 4055 and independent prognostic relevance was found only for advanced Gleason grade, high tumor stage, high preoperative PSA level, positive nodal status and positive surgical margin status $(P<0.0001$ each) but not for KPNA2 immunostaining $(P=0.059)$. Multivariate analysis including only criteria available before surgery, such as maximal Gleason grade on biopsies, clinical stage and preoperative PSA level, again revealed a strong independent prognostic relevance for advanced biopsy Gleason grade, advanced clinical stage, high PSA level and also KPNA2 expression $(P<0.0001$ each). For all these statistical calculations, all variables (including those available before and after surgery) were used in the same categories as shown in Table 1. 


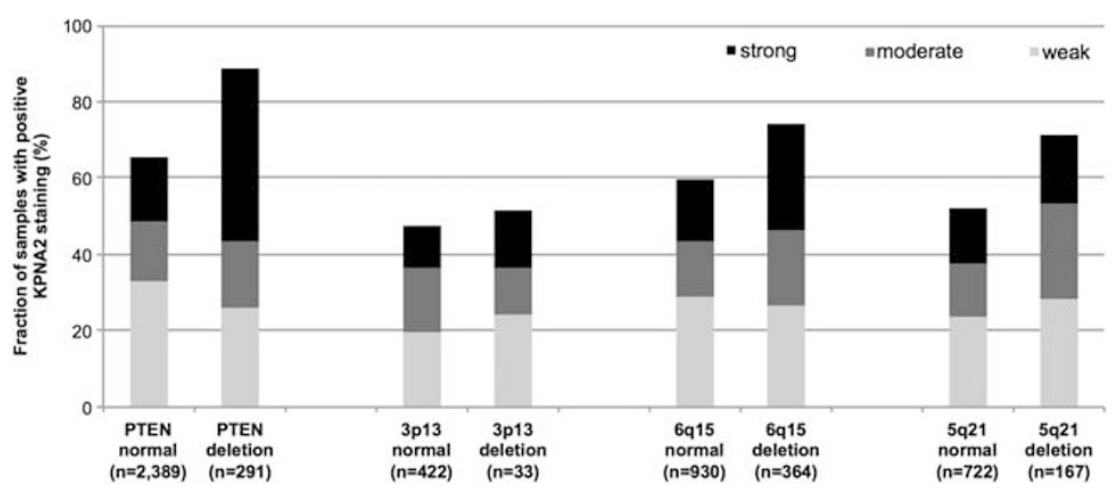

Figure 4 KPNA2 expression vs deletions at PTEN $(P<0.0001)$, 3p13 $(P=0.7514)$, 6q15 $(P<0.0001)$ and $5 q 21(P<0.0001)$ probed by fluorescence in situ hybridization analysis in ERG-negative cancers.

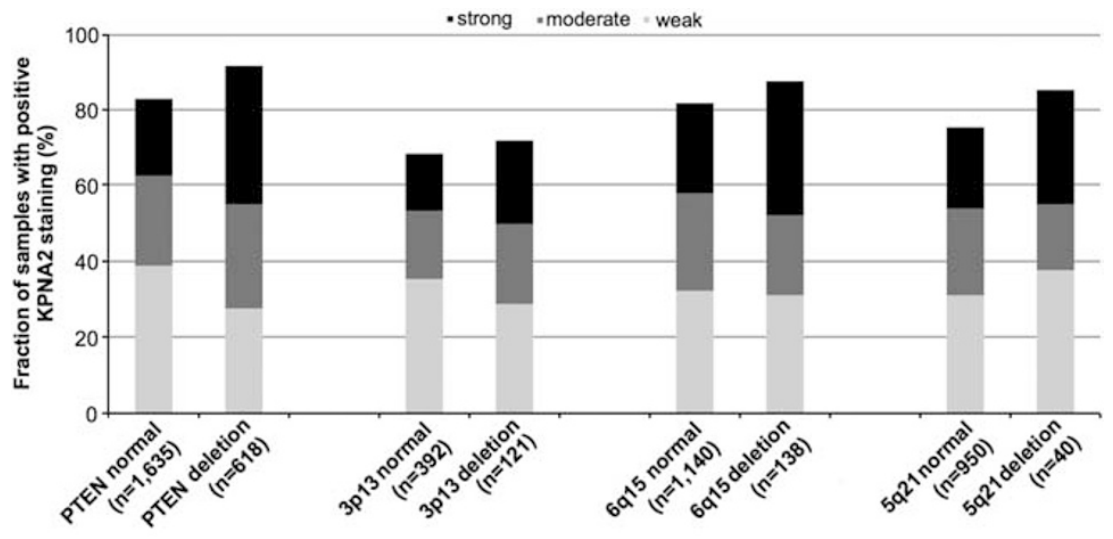

Figure 5 KPNA2 expression vs deletions at PTEN $(P<0.0001)$, 3p13 $(P=0.1622)$, $6 \mathrm{q} 15(P=0.027)$ and $5 \mathrm{q} 21(P=0.2662)$ probed by fluorescence in situ hybridization analysis in ERG-positive cancers.

\section{Discussion}

The results of our study identify KPNA2 expression as a potentially clinically useful prognostic marker for prostate cancer.

Immunohistochemical analyses revealed positive nuclear KPNA2 staining in $68.3 \%$ of 7964 interpretable prostate cancers treated by radical prostatectomy. One earlier study performing KPNA2 IHC on a prostate cancer TMA described nuclear KPNA2 expression in $47 \%$ of 606 interpretable prostate cancers. ${ }^{4}$ The slightly divergent results between this and our study are most likely due to differences in IHC protocols and scoring systems. For example, a rabbit antibody from Abcam was used for KPNA2 detection in our study, whereas Mortezavi et al ${ }^{4}$ used a primary goat antibody from Santa Cruz Biotechnology. Also, the scoring system varied markedly. In the study of Mortezavi et $a l,{ }^{4}$ the median nuclear KPNA2 immunoreactivity in prostatectomy cases (median $0 \%$ ) was chosen as cutoff, and accordingly, positive nuclear KPNA2 immunoreactivity was defined as nuclear staining in at least $0.1 \%$ of target cells. In our study, an established predefined score was used that was built for each tissue spot from the two parameters, the staining intensity $(0,1+, 2+$ and $3+)$ and the fraction of positive tumor cells. ${ }^{23-25}$

Our scoring system is based on the fact that the staining intensity is less reliable than the fraction of stained tumor cells. It is important to note that fixation-related variations of the staining intensity are an inherent feature of IHC experiments. For example, a $2+$ staining intensity can indicate true medium-level protein expression under optimal fixation conditions, but might as well result from 'understaining' of high-level expression in case of suboptimal fixation. In contrast, the fraction of stained tumors cells is virtually not affected by the fixation quality. Our scoring system was defined to accomodate these facts. For example, a $1+$ cancer with $70 \%$ stained tumor cells is considered 'moderate' because we consider the high fraction of stained cells more important than the low staining intensity, which might be due to poor immunoreactivity. Vice versa, a $2+$ cancer with $\leq 30 \%$ stained cells is considered 'weak', as it has only a small fraction of positive tumors cells, although the tissue sample was probably optimally immunoreactive. However, the thresholds of $30-70 \%$ were selected almost arbitrarily based on the experience that they provide solid results in terms of detecting 

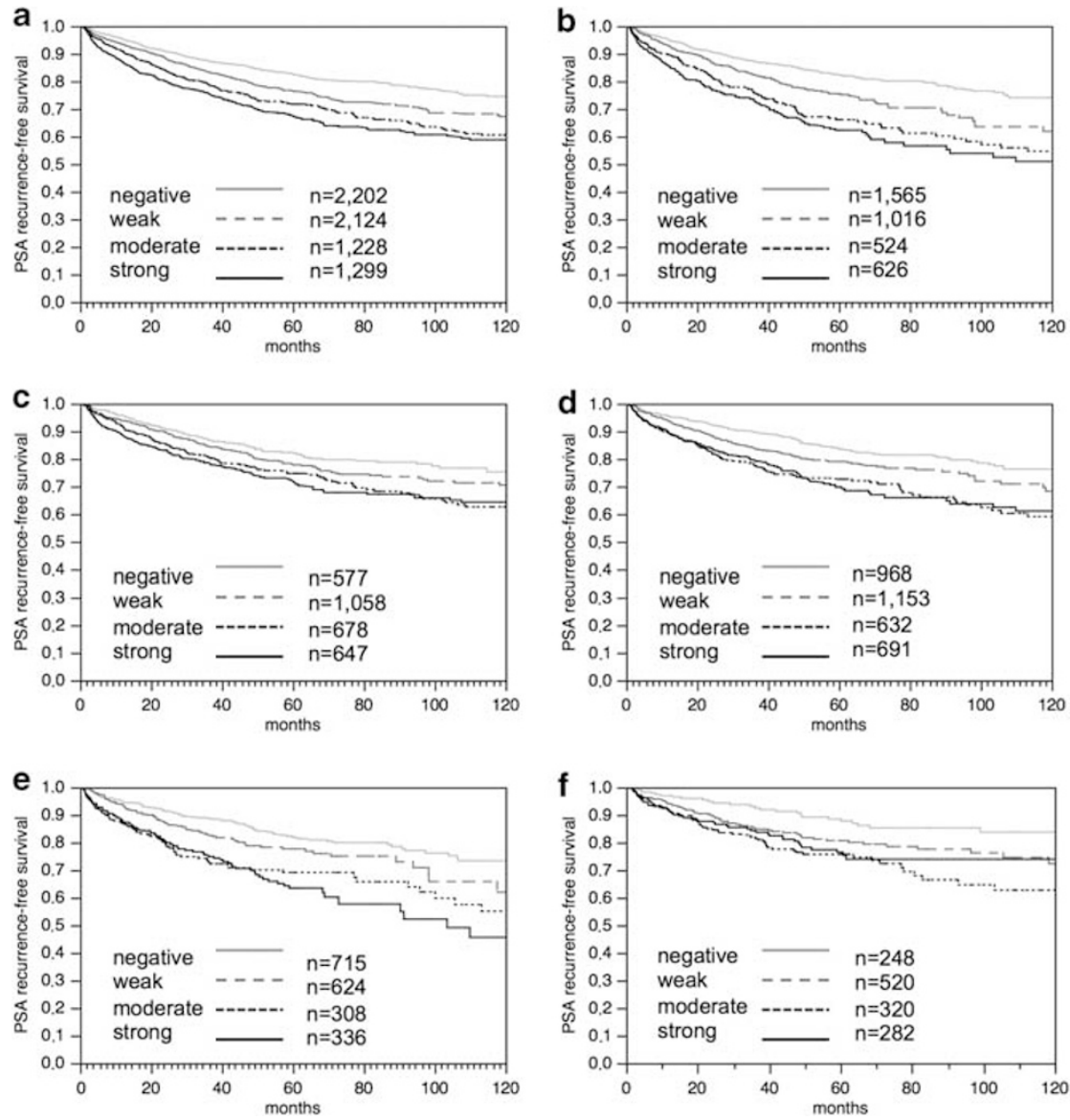

Figure 6 Relationship of KPNA2 immunostaining intensity with biochemical recurrence in (a) all cancers $(n=6853 ; P<0.0001)$, (b) ERGnegative cancers $(n=3731 ; P<0.0001)$, (c) ERG-positive cancers $(n=2960 ; P<0.0001)$, (d) $P T E N$-non-deleted cancers $(n=3372$; $P<0.0001)$, (e) $P T E N$-non-deleted ERG-negative cancers $(n=1983 ; P<0.0001)$, and (f) $P T E N$-non-deleted ERG-positive cancers $(n=1370$; $P=0.0009)$. PSA, prostate-specific antigen.

significant associations between molecular markers and clinicopathological tumor features in many previous studies. ${ }^{23-25}$ Thus, these findings are yet another example of the robustness of the TMA technology for the identification of genotype/ phenotype associations. Using our prostate cancer TMA, we have previously demonstrated the prognostic relevance of several established and new molecular markers in prostate cancer, including

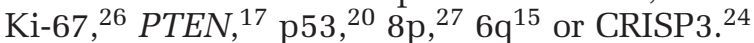

The strong association of high-level KPNA2 staining with early PSA recurrence represents the most significant finding of this study. That the prognostic nature of high KPNA2 expression levels was independent of powerful prognostic factors such as Gleason grade, tumor stage and surgical margin status (Figure 7) is remarkable, especially since KPNA2 expression was only analyzed in a minute tissue specimen measuring $0.6 \mathrm{~mm}$ per tumor.

It has been suggested before that the analysis of multiple cores per tumor specimen could improve the representativity of TMA studies. ${ }^{28}$ This recommendation was based on a better concordance of large section findings with TMA data, if 3-4 cores were utilized per cancer as compared with the use of only one sample. However, these findings were based on the assumption that significant heterogeneity may exist within the tissue represented by a standard $3 \times 4 \mathrm{~cm}^{2}$ paraffin block, and that large section analysis is the method of choice to estimate tumor heterogeneity. In our opinion, this assumption is highly disputable. We have shown earlier that the TMA format is generally superior over large section studies to reveal associations between molecular markers and the clinical course. In this study, we compared TMA and large section findings of p53, ER and PR in breast cancer, and demonstrated that overinterpretation of focal p53 positivity in large sections obscured the established prognostic relevance of p53, which was, however, perfectly found in the TMA format. ${ }^{29}$ More recently, we extended these analyses to prostate cancer and studied the impact of the number of cores on the prognostic impact of $\mathrm{Ki}-67$ and p53 in prostate cancer in detail. ${ }^{30}$ In this study, 1-3 cores of a subset $(n=3261)$ of the tumors of our current prostate cancer TMA was analyzed. The results revealed that the same significant associations between Ki-67 or p53 expression and increased tumor stage high Gleason grade or early PSA are found irrespective 

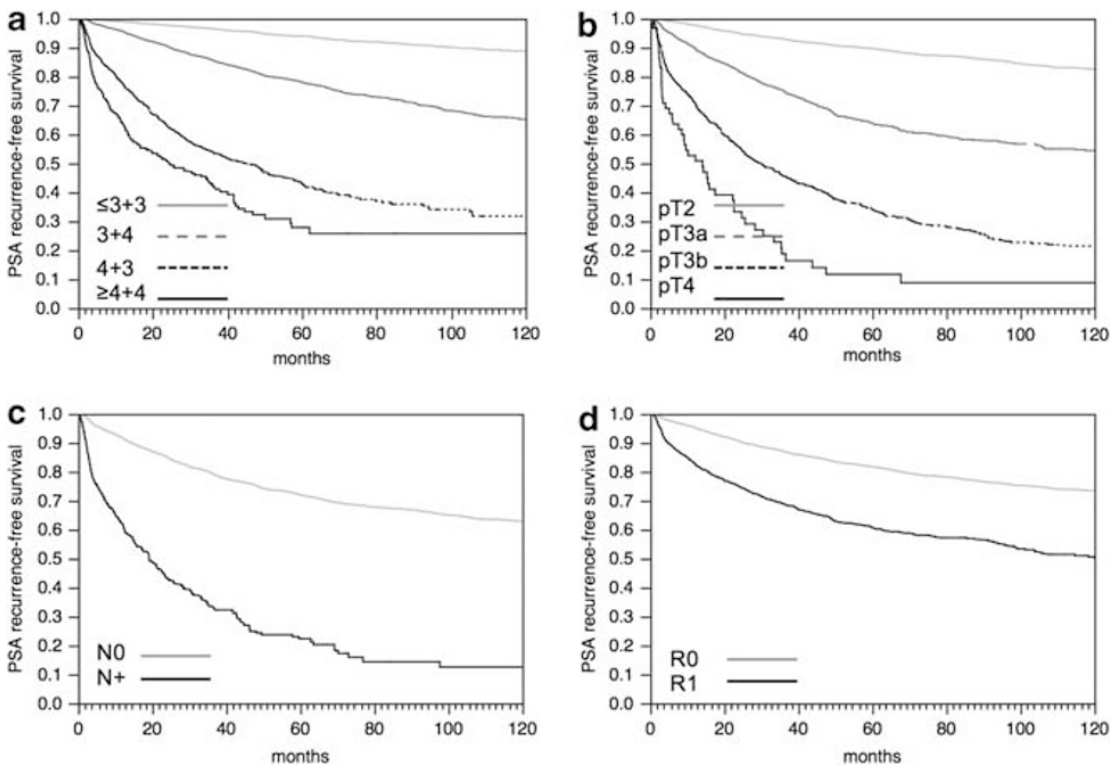

Figure 7 Relationship of biochemical recurrence with (a) Gleason grade, (b) tumor stage, (c) lymph nodal status and (d) surgical margin status $(P<0.0001$ each). PSA, prostate-specific antigen.

of whether the three tissue cores were analyzed separately or a combined result was generated from the three cores. These data demonstrate that the use of multiple cores in a TMA does not necessarily increase the ability to identify associations of biomarkers with tumor phenotype and prognosis. Lately, we introduced our 'heterogeneity TMA platform' for identification of intra- and interfocal heterogenity of ERG fusion in prostate cancer. ${ }^{31}$ The rational of this TMA format was based on the hypothesis that multiple subclones arise during cancer progression, and that these subclones may only be identified when the entire tumor bulk is analyzed rather than a single, preselected tumor block. For this study, we manufactured a TMA from the radical prostatectomy specimens of 190 patients. Each prostate was completely dissected, and all tissue blocks were inspected for the presence of cancer. Tissue punches were taken from 10 different remote tumor-containing blocks of each prostate, and all 1900 tissue punches were assembled in one TMA. Each prostate was also inspected for the presence of multiple independent tumor foci according to the established criteria. ${ }^{32}$ In brief, the results of this study showed that ERG expression was frequently $(42 \%$ of patients) heterogeneous across the 10 cores, whereas only $16 \%$ of patients had tumors with homogeneous ERG positivity. ERG heterogeneity was within one tumor focus (intrafocal heterogeneity) in 69 cases $(93 \%$ of heterogeneous cases) and between different tumor foci (interfocal heterogeneity) in 5 cases (7\%). Importantly, heterogeneity within one tissue block was found only in $4(0.5 \%)$ tissue spots out of $>700$ ERG-positive tissue spots from 103 different patients, with the findings of the TMA cores being validated by large section analysis in 14 cases.
Taken together, the results of these studies clearly demonstrate that TMAs are superior to large section studies for identifying clinically relevant associations, that multiple cores taken from the same donor block do not increase the power of such analyses and that relevant heterogenity (ie, coexistence of ERG-negative and -positive tumor subclones) can only be reliably identified if the entire tumor bulk is analyzed, but not if the analysis is limited to the small piece of tumor typically represented in a standard $3 \times 4 \mathrm{~cm}^{2}$ tissue block.

In line with our results, Mortezavi et al ${ }^{4}$ had also suggested KPNA2 expression as an independent prognosticator for biochemical recurrence. From these data it appears possible that KPNA2 expression may serve as an even more powerful prognostic biomarker in a preoperative setting where definitive tumor stage, Gleason grade and surgical margin status are unavailable. If molecular analyses are applied on core needle biopsies, the quantity of analyzable cancer tissue is comparable to a TMA analysis (or even larger). In an attempt to model a preoperative scenario, we had indeed found an even stronger independent impact of KPNA2 staining if analyzed together with preoperative PSA and biopsy Gleason grade. It is worth noting that the Gleason grading has substantial limitations if determined on biopsies. The Gleason grade as determined on core needle biopsies reflects the final Gleason grade obtained on the resection specimen in only about $65 \%$ with undergrading representing the most frequent problem on needle biopsies. ${ }^{33}$ It appears likely that molecular features such as KPNA2 measurement will be even more helpful in settings with suboptimal histology data.

The biological function of KPNA2 is consistent with a significant role for cancer development and 
progression. Karyopherins mediate the nuclear import of various factors with relevance for prostate cancer such as androgen receptor, MYC, BCAR1 and p53. ${ }^{34-37}$ Accordingly, functional analyses on cell lines have suggested an oncogenic function of KPNA2 overexpression in promoting proliferation, viability and migration of cancer cells. ${ }^{4-7}$ Recent studies linking high KPNA2 expression to adverse clinical outcome in breast $^{8}$ and ovarian cancer, ${ }^{10}$ melanoma, ${ }^{9}$ and astrocytoma ${ }^{11}$ provide further clinical evidence for a strong role of KPNA2 in cancer biology. That aberrations in molecules regulating nuclear import of tumorrelevant proteins can have a pivotal role in cancer cells is also supported by the recent observation that overexpression of Ran, another important protein of the nuclear import machinery, is linked to adverse tumor phenotype in breast and lung cancers. ${ }^{38}$

A further aim of this study was to analyze whether KPNA2 expression is linked to key genomic alterations in prostate cancers by taking advantage of our TMA-attached database. About half of prostate cancers carry gene fusions linking the androgenregulated gene TMPRSS2 with transcription factors of the ETS family. ${ }^{39}$ As a result of this rearrangement, $E R G$ becomes androgen regulated and massively overexpressed. Our data demonstrate that KPNA2 expression is significantly associated with positive $E R G$ status. Finding this association by two independent approaches for $E R G$ fusion detection (IHC/FISH) largely excludes a falsepositive association due to inefficient immunostaining for both KPNA2 and ERG in a subset of damaged non-reactive tissues. Activation of ERG expression in prostate epithelial cells results in aberrant activation of different signaling cascades involved in the regulation of cell adhesion, matrix remodeling and signal transduction. ${ }^{40-43}$ It is tempting to speculate that the higher KPNA2 expression in ERG-positive than in ERG-negative cancers may be driven by an increased requirement for nuclear transportation capacity in these tumors. For example, several signaling cascades, such as the WNT and TGF- $\beta$ pathways, which are known to be activated by ERG, ${ }^{40,41,44}$, rely on efficient nuclear import of their effector molecules, including $\beta$-catenin and SMAD. ${ }^{45,46}$

Further subgroup analyses involved tumor populations defined by genomic deletions that are known to be tightly linked to the $E R G$ status. In particular, deletions at 3p13 and PTEN occur in ERG-positive and deletions of 5q21 and 6q15 in ERG-negative prostate cancers. ${ }^{12-19}$ Interestingly, KPNA2 staining was tightly linked to PTEN and 6q15 deletions in both ERG-negative and -positive cancers. These findings might suggest that either activation of a pathway that also induces strong KPNA2 expression may facilitate PTEN and 6q15 inactivation, or PTEN and $6 q 15$ inactivation may facilitate the development of certain molecular features, eventually leading to strong KPNA2 expression. Evidence for the latter hypothesis comes from the established functional relationship between PTEN deletion and increased PI3K/AKT signaling, ${ }^{47}$ which has been shown to enhance both the activity and capacity of the nucleocytoplasmic transportation machinery. ${ }^{48,49}$

The existence of rearrangements involving alternative ETS family members in 'fusion-type' prostate cancer, which are undetectable by ERG IHC, offers an alternative explanation for the strong association of PTEN loss with KPNA2 expression in ERGnegative cancers. PTEN deletions are known to be very tightly linked to ERG-positive cancer. ${ }^{50}$ In our cohort, $29.1 \%$ of ERG-positive but only $10.7 \%$ of ERG-negative cancers are PTEN deleted. ${ }^{17}$ Although experimental proof is lacking, one could speculate that PTEN deletions are also markedly increased in these $5-10 \%$ of 'fusion-type' prostate cancers having alternate rearrangements not leading to ERG overexpression. If so, the strong association of KPNA2 overexpression with fusion-type prostate cancer could cause the observed association of KPNA2 and PTEN deletions in ERG-negative cancers.

In summary, our study identified a strong link of high KPNA2 expression with early PSA recurrence, which was independent of Gleason grade, tumor stage, margin status and preoperative PSA level in a series of more than 6000 interpretable cancers. The even stronger independent prognostic impact of KPNA2 expression in a setting using only parameters that are preoperatively available raises the possibility that measurement of KPNA2 expression alone or in combination with other molecular features might result in clinically useful information.

\section{Acknowledgements}

We thank Christina Koop, Julia Schumann, Sünje Seekamp and Inge Brandt for excellent technical assistance.

\section{Disclosure/conflict of interest}

The authors declare no conflict of interest.

\section{References}

1 Jemal A, Bray F, Center MM, et al. Global cancer statistics. CA Cancer J Clin 2011;61:69-90.

2 Nakielny S, Dreyfuss G. Transport of proteins and RNAs in and out of the nucleus. Cell 1999;99: 677-690.

3 Teng SC, Wu KJ, Tseng SF, et al. Importin KPNA2, NBS1, DNA repair and tumorigenesis. J Mol Histol 2006;37:293-299.

4 Mortezavi A, Hermanns T, Seifert HH, et al. KPNA2 expression is an independent adverse predictor of biochemical recurrence after radical prostatectomy. Clin Cancer Res 2011;17:1111-1121. 
5 Noetzel E, Rose M, Bornemann J, et al. Nuclear transport receptor karyopherin-alpha2 promotes malignant breast cancer phenotypes in vitro. Oncogene 2012;31:2101-2114.

6 Umegaki N, Tamai K, Nakano H, et al. Differential regulation of karyopherin alpha 2 expression by TGFbeta1 and IFN-gamma in normal human epidermal keratinocytes: evident contribution of KPNA2 for nuclear translocation of IRF-1. J Invest Dermatol 2007;127:1456-1464.

7 Wang CI, Wang CL, Wang CW, et al. Importin subunit alpha-2 is identified as a potential biomarker for nonsmall cell lung cancer by integration of the cancer cell secretome and tissue transcriptome. Int J Cancer 2011;128:2364-2372.

8 Gluz O, Wild P, Meiler R, et al. Nuclear karyopherin alpha2 expression predicts poor survival in patients with advanced breast cancer irrespective of treatment intensity. Int J Cancer 2008;123:1433-1438.

9 Winnepenninckx V, Lazar V, Michiels S, et al. Gene expression profiling of primary cutaneous melanoma and clinical outcome. J Natl Cancer Inst 2006;98:472-482.

10 Zheng M, Tang L, Huang L, et al. Overexpression of karyopherin-2 in epithelial ovarian cancer and correlation with poor prognosis. Obstet Gynecol 2010;116:884-891.

11 Gousias K, Becker AJ, Simon M, et al. Nuclear karyopherin a2: a novel biomarker for infiltrative astrocytomas. J Neurooncol 2012;109:545-553.

12 Lapointe J, Li C, Giacomini CP, et al. Genomic profiling reveals alternative genetic pathways of prostate tumorigenesis. Cancer Res 2007;67:8504-8510.

13 Taylor BS, Schultz N, Hieronymus H, et al. Integrative genomic profiling of human prostate cancer. Cancer Cell 2010;18:11-22.

14 Berger MF, Lawrence MS, Demichelis F, et al. The genomic complexity of primary human prostate cancer. Nature 2011;470:214-220.

15 Kluth M, Hesse J, Heinl A, et al. Genomic deletion of MAP3K7 at 6q12-22 is associated with early PSA recurrence in prostate cancer and absence of TMPRSS2:ERG fusions. Mod Pathol 2013;26:975-983.

16 Burkhardt L, Fuchs S, Krohn A, et al. CHD1 is a 5q21 tumor suppressor required for ERG rearrangement in prostate cancer. Cancer Res 2013;73:2795-2805.

17 Krohn A, Diedler T, Burkhardt L, et al. Genomic deletion of PTEN is associated with tumor progression and early PSA recurrence in ERG fusion-positive and fusionnegative prostate cancer. Am J Pathol 2012;181:401-412.

18 Huang S, Gulzar ZG, Salari K, et al. Recurrent deletion of CHD1 in prostate cancer with relevance to cell invasiveness. Oncogene 2012;31:4164-4170.

19 Liu S, Yoshimoto M, Trpkov K, et al. Detection of ERG gene rearrangements and PTEN deletions in unsuspected prostate cancer of the transition zone. Cancer Biol Ther 2011;11:562-566.

20 Schlomm $\mathrm{T}$, Iwers $\mathrm{L}$, Kirstein $\mathrm{P}$, et al. Clinical significance of p53 alterations in surgically treated prostate cancers. Mod Pathol 2008;21:1371-1378.

21 Mirlacher M, Simon R. Recipient block TMA technique. Methods Mol Biol 2010;664:37-44.

22 Minner S, Enodien M, Sirma H, et al. ERG status is unrelated to PSA recurrence in radically operated prostate cancer in the absence of antihormonal therapy. Clin Cancer Res 2011;17:5878-5888.

23 Minner S, Wittmer C, Graefen M, et al. High level PSMA expression is associated with early PSA recurrence in surgically treated prostate cancer. Prostate 2011;71:281-288.
24 Grupp K, Kohl S, Sirma H, et al. Cysteine-rich secretory protein 3 overexpression is linked to a subset of PTEN-deleted ERG fusion-positive prostate cancers with early biochemical recurrence. Mod Pathol 2013; 26:733-742.

25 Muller J, Ehlers A, Burkhardt L, et al. Loss of pSer2448-mTOR expression is linked to adverse prognosis and tumor progression in ERG-fusion-positive cancers. Int J Cancer 2013;132:1333-1340.

26 Bubendorf L, Sauter G, Moch H, et al. Ki67 labelling index: an independent predictor of progression in prostate cancer treated by radical prostatectomy. J Pathol 1996;178:437-441.

27 El Gammal AT, Bruchmann M, Zustin J, et al. Chromosome $8 p$ deletions and $8 \mathrm{q}$ gains are associated with tumor progression and poor prognosis in prostate cancer. Clin Cancer Res 2010;16:56-64.

28 Rubin MA, Dunn R, Strawderman M, et al. Tissue microarray sampling strategy for prostate cancer biomarker analysis. Am J Pathol 2002;26: 312-319.

29 Torhorst J, Bucher C, Kononen J, et al. Tissue microarrays for rapid linking of molecular changes to clinical endpoints. Am J Pathol 2001;159:2249-2256.

30 Tennstedt P, Koster P, Bruchmann A, et al. The impact of the number of cores on tissue microarray studies investigating prostate cancer biomarkers. Int J Oncol 2012;40:261-268.

31 Minner S, Gartner M, Freudenthaler F, et al. Marked heterogeneity of ERG expression in large primary prostate cancers. Mod Pathol 2013;26:106-116.

32 Wise AM, Stamey TA, McNeal JE, et al. Morphologic and clinical significance of multifocal prostate cancers in radical prostatectomy specimens. Urology 2002;60: 264-269.

33 Epstein JI, Feng Z, Trock BJ, et al. Upgrading and downgrading of prostate cancer from biopsy to radical prostatectomy: incidence and predictive factors using the modified Gleason grading system and factoring in tertiary grades. Eur Urol 2012;61:1019-1024.

34 Cutress ML, Whitaker HC, Mills IG, et al. Structural basis for the nuclear import of the human androgen receptor. J Cell Sci 2008;121:957-968.

35 Kim IS, Kim DH, Han SM, et al. Truncated form of importin alpha identified in breast cancer cell inhibits nuclear import of p53. J Biol Chem 2000;275: 23139-23145.

$36 \mathrm{Li} \mathrm{S}, \mathrm{Ku}$ CY, Farmer AA, et al. Identification of a novel cytoplasmic protein that specifically binds to nuclear localization signal motifs. J Biol Chem 1998;273: 6183-6189.

37 Nadler SG, Tritschler D, Haffar OK, et al. Differential expression and sequence-specific interaction of karyopherin alpha with nuclear localization sequences. J Biol Chem 1997;272:4310-4315.

38 Yuen HF, Chan KK, Grills C, et al. Ran is a potential therapeutic target for cancer cells with molecular changes associated with activation of the PI3K/Akt/ mTORC1 and Ras/MEK/ERK pathways. Clin Cancer Res 2012;18:380-391.

39 Tomlins SA, Rhodes DR, Perner S, et al. Recurrent fusion of TMPRSS2 and ETS transcription factor genes in prostate cancer. Science 2005;310:644-648.

40 Brase JC, Johannes M, Mannsperger $\mathrm{H}$, et al. TMPRSS2-ERG-specific transcriptional modulation is associated with prostate cancer biomarkers and TGF-beta signaling. BMC Cancer 2011;11:507. 
41 Gupta S, Iljin K, Sara H, et al. FZD4 as a mediator of ERG oncogene-induced WNT signaling and epithelialto-mesenchymal transition in human prostate cancer cells. Cancer Res 2010;70:6735-6745.

42 Hermans KG, Boormans JL, Gasi D, et al. Overexpression of prostate-specific TMPRSS2(exon 0)-ERG fusion transcripts corresponds with favorable prognosis of prostate cancer. Clin Cancer Res 2009;15:6398-6403.

43 Tomlins SA, Laxman B, Varambally S, et al. Role of the TMPRSS2-ERG gene fusion in prostate cancer. Neoplasia 2008;10:177-188.

44 Jhavar S, Brewer D, Edwards S, et al. Integration of ERG gene mapping and gene-expression profiling identifies distinct categories of human prostate cancer. BJU Int 2009;103:1256-1269.

45 Cadigan KM, Peifer M. Wnt signaling from development to disease: insights from model systems. Cold Spring Harb Perspect Biol 2009;1:a002881.
46 Schmierer B, Hill CS. TGFbeta-SMAD signal transduction: molecular specificity and functional flexibility. Nat Rev Mol Cell Biol 2007;8:970-982.

47 Maehama T, Dixon JE. The tumor suppressor, PTEN/ MMAC1, dephosphorylates the lipid second messenger, phosphatidylinositol 3,4,5-trisphosphate. J Biol Chem 1998;273:13375-13378.

48 Ly TK, Wang J, Pereira R, et al. Activation of the Ran GTPase is subject to growth factor regulation and can give rise to cellular transformation. J Biol Chem 2010; 285:5815-5826.

49 Radisavljevic ZM, Gonzalez-Flecha B. TOR kinase and Ran are downstream from PI3K/Akt in H2O2-induced mitosis. J Cell Biochem 2004;91:1293-1300.

50 Carver BS, Tran J, Gopalan A, et al. Aberrant ERG expression cooperates with loss of PTEN to promote cancer progression in the prostate. Nat Genet 2009;41: $619-624$. 